\title{
EELS Measurements on Wurtzite InN
}

\author{
K. A. Mkhoyan*, E. S. Alldredge**, N. W. Ashcroft**, J. Silcox* \\ *School of Applied and Engineering Physics, Cornell University, Ithaca NY 14853 \\ **Physics Department, Cornell University, Ithaca NY 14853
}

Recent successes in the growth of high quality wurtzite $\operatorname{InN}^{1}$ have stimulated interest in the use of InN for high performance high electron mobility transistors (HEMTS) or light-emitting diodes. ${ }^{2}$ However, unlike other III-V nitrides such as GaN or AlN, there is a dearth of reliable data on the basic structural, electronic and optical parameters and properties of $\mathrm{InN}$.

Electron energy loss spectroscopy (EELS) is a powerful technique to measure some of these properties. Here we report EELS measurements on wurtzite InN using the Cornell UHV VG HB501 scanning transmission electron microscope (STEM) with a $100 \mathrm{keV}$ electron beam and compare these with theoretical band structure calculations relevant to the observations.

The excitations of the $\mathrm{N} 1 s$ core-level electrons to the empty $\mathrm{N} 2 p$ states of conduction band give rise to the characteristic N K-edge spectrum in EELS which is presented in Fig. 1 where the positions of featuring peaks are identified. The dashed line (Fig. 1) is the calculated (using density functional theory within the local density approximation) $\mathrm{N} 2 p$ partial density of states in the conduction band of the InN. There is excellent agreement here on the relative position of the peaks. For In $M_{4,5}$-edge three broad peaks, $P_{1}-P_{3}$ at about $472 \mathrm{eV}, 479 \mathrm{eV}$ and $488-490 \mathrm{eV}$, are identified. The results of Xray photoemission spectroscopy measurements ${ }^{3}$ suggest that the primary contributions here in EELS come from transitions of the electrons from In $3 d_{3 / 2}$ and $3 d_{5 / 2}$ states to the corresponding In $5 p$ empty states of conduction band. In a simple model, we convolved the spectrum of the In $3 d_{3 / 2}$ and $3 d_{5 / 2}$ states (see Fig. 2(b)) with the calculated partial, In 5p, conduction band DOS (Fig. 2(c)) which explains the presence of peaks $P_{1}-P_{3}$.

The next step was the study of the low-loss region of the EELS in InN. After Fourier-Log deconvolutions, the resulting single inelastic scattering distribution (SSD) is presented in Fig. 3. The strong peak located at $15.5 \pm 0.1 \mathrm{eV}$ corresponds to this plasmon-loss. Calculations of the band structure predicts, as in the case of $\mathrm{GaN}$, the presence of well-defined In $4 d$ deep valence states in InN. Photoelectronic spectroscopy measurements ${ }^{3}$ carried out in InN confirms the existence of In $4 d$ states in InN. In low-loss EELS an inelastic interaction of the beam electrons with electrons of In $4 d$ states causes transitions of the latter into unoccupied states of the conduction band where the dipole selection rule eliminates all transitions except those into In $5 p$ empty states. Calculated In $5 p$ partial DOS of the conduction band convoluted with a simple Gaussian function for $4 d$ states is also presented in Fig. 3 (dashed line). The first peak of the DOS curve is aligned with peak A at 20.4 \pm 0.1 $\mathrm{eV}$. As can be seen it describes the existence of peaks A-C in low-loss EELS (the broad peaks B and $\mathrm{C}$ are located within 23-25 and 34-40 eV ranges respectively). This predicts that, if we take into account the $1.9 \mathrm{eV}$ band gap of $\mathrm{InN}$, the In $4 d$ valence states are located $14.4 \pm 0.5 \mathrm{eV}$ below the top of the valence band.

\section{Refereces}

[1] H. Lu, W.J. Schaff, J. Hwang, H. Wu, G. Koley, L.F. Eastman, Appl. Phys. Lett.79, 1489 (2001). 
[2] S. Strite and H. Morkoc, J. Vac. Sci. Technol. B 10, 1237 (1992).

[3] Q.X. Guo, M. Nishio, H. Ogawa, A. Wakahara, A. Yoshida, Phys. Rev. B 58, 15304 (1998).

[4] This work is supported by Office of Naval Research Contract No. N00014-99-10714, under the direction of Dr. C. E. C. Wood.
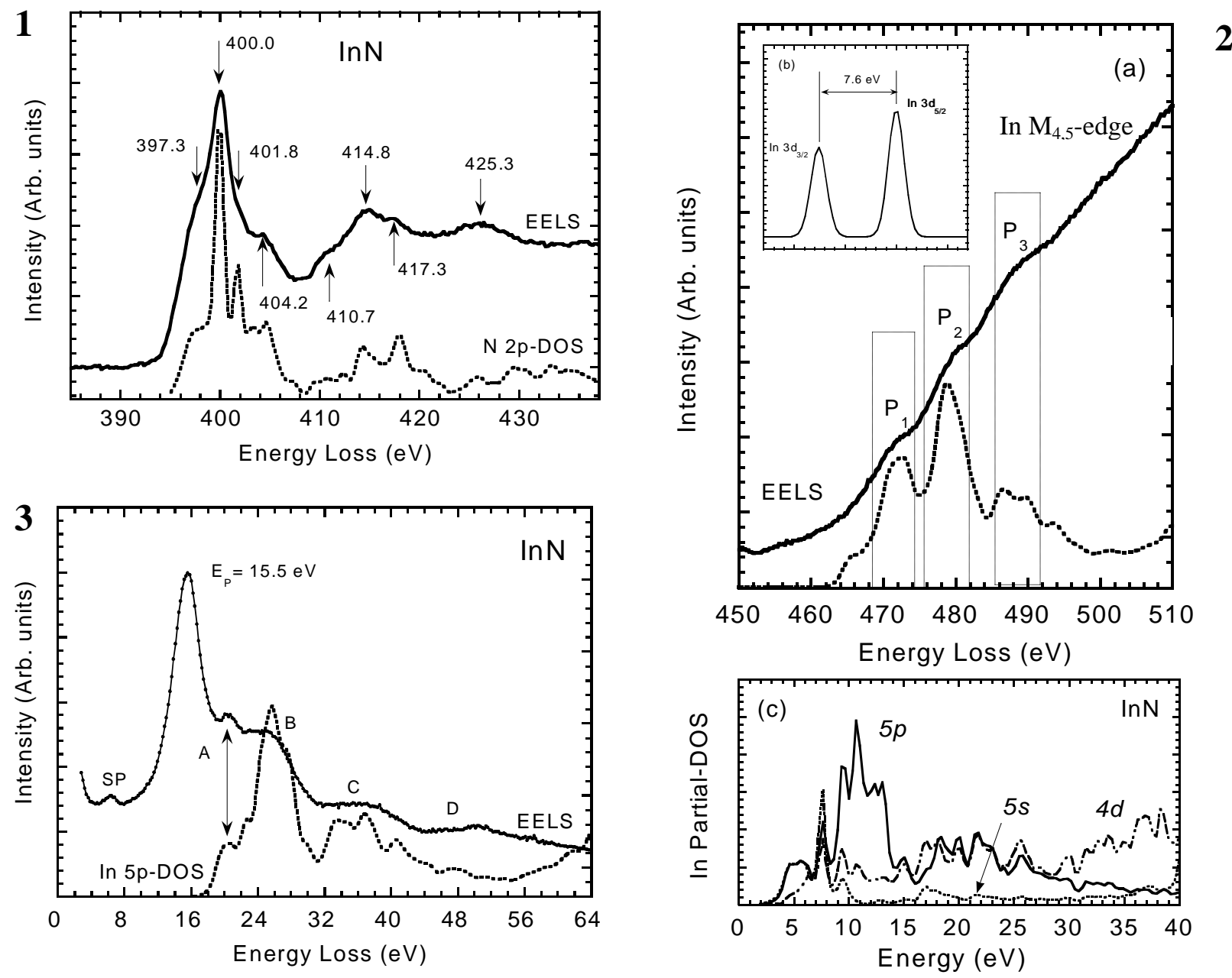

Fig. 1. Nitrogen $K$-edge from EELS measurements in wurtzite InN (solid line) with calculated nitrogen N $2 p$ partial DOS (dashed line). The DOS calculations are aligned to EELS by displacing the primary peak to $400.0 \mathrm{eV}$ for better comparison of the remaining features.

Fig. 2. In $M_{4,5}$-edge from EELS measurements in wurtzite InN (solid line) with its theoretical prediction (dashed line) obtained by convoluting In $3 d_{3 / 2}$ and $3 d_{5 / 2}$ states (from Ref. 3) (b) with corresponding In $5 p$ empty conduction band DOS. (c) partial, In $5 s, 5 p$ and $4 d$, DOS of the conduction band.

Fig. 3. Single scattering distribution obtained from low-loss EELS. The dashed line is calculated In $5 p$ partial DOS of the conduction band convoluted with In $4 d$ valence states. 